\title{
Influence of Machining Parameters on Vibration Characteristics of Gear Form Grinding
}

\author{
Huiliang Wang ${ }^{1,2^{*}}$, Yangqi $\mathrm{CaO}^{1 * *}$ \\ ${ }^{1}$ School of Mechatronics Engineering, Henan University of Science and Technology, Luoyang, China \\ ${ }^{2}$ Collaborative Innovation Center of Machinery Equipment Advanced Manufacturing, Luoyang, China \\ E-mail: whl-ly@163.com*, angel725@vip.qq.com**
}

\begin{abstract}
Based on the geometry theory and kinematics principle of gear form grinding machine, a mathematical model of gear form grinding machine vibration is established. Using the multiple scales method, the model is analyzed and solved, which provides a theoretical basis for the system study of the relationship between natural frequency and external excitation frequency. According to the theoretical analysis results, the test platform is set up on the YK7660 gear form grinding machine. By varying the parameters of grinding process, the data of vibration characteristics of gear form grinding is acquired and analyzed. The experimental results show that the change of grinding depth has great influence on the grinding force; meanwhile when the cutting depth is increased from $0.01 \mathrm{~mm}$ to $0.02 \mathrm{~mm}$, the $X$ axis vibration amplitude increases by $19 \%$ compared with the feed rate of the workpiece. The research of vibration characteristics of gear form grinding machine proves the validity of the grinding force derived by vibration theory, which provides the theoretical foundation for improving the accuracy of grinding gear in practical application.
\end{abstract}

Keywords: Gear form grinding, multiple scales method, machining parameters, vibration characteristics.

\section{Introduction}

With the development of high speed and heavy load gear transmission, more and more people pay attention to the accuracy of gears. At present, the widely used processing methods in gear machining are generating and forming process which can be a very effective means for eliminating tooth flank errors [13]. Compared with the generating process, in the machining of high precision gears, forming process will play an important role in the future with the advantages of high efficiency, high precision, and the distinguished capacity of machining gears with some special requirements [4-9]. However, when the grinding machines work for a long time, their mechanical characteristics will be degraded, such as structure, gap, static stiffness, vibration etc., which makes their mechanical properties change, and then affects the machining precision of gear.

In the grinding process, Orynski [10] got the change tendency of damping parameters affecting the vibration stability factors by establishing, solving, and analyzing the mathematical model of grinding process. Krajnik et al. [11] made a comparative analysis of the centerless grinding using the nonlinear method and genetic algorithm. Toenshof [12-13] established the mathematical model of grinding process, which obtained the unstable growth factor and stability limit. Hahimoto et al. [14-15] analyzed the factors affecting stability of system by using the theoretical and experimental analysis on the equation of dynamic interference between grinding wheel and workpiece. Inasdki et al. [16] studied the stability of grinding system and unstable growth limit coefficient by exploring the roots of the characteristic equation of grinding process. According to the grinding wheel regenerative chatter and workpiece regenerative chatter, Weck [17] and Matsubara [18] respectively studied the stability of a system on dynamic process. Ebrahimi and Whalley [19] described a concept of machine process modeling for virtual machining purposes; Wang et al. [20] proposed a method for quantitative prediction of vibration gearbox using gray bootstrap theory and verified by experiment. However, within related research, although their methods for vibration characteristic provided a development of gear form grinding machine, the effect degree of technological parameters on vibration characteristics of each servo shaft was not pointed out. 
This work aims to analyze the relationship among the grinding process parameters, the grinding force and vibration by collecting the acceleration signal of each servo shaft and the current signal of the electric spindle signals in the process of grinding gear. The analysis of the results will contribute to improving the gear processing precision. The outline of the remainder of the paper is as follows. First, we establish a mathematical model of gear form grinding machine vibration characteristics. Subsequently, using multiple scales method, the equation of primary resonance is solved. These contents constitute the second chapter. Next, vibration test of gear form grinding machine is introduced in Chapter 3. Then, the effect of vibration characteristics under different parameters on each servo shaft is illustrated in Chapter 4. Finally, some conclusions are drawn in Chapter 5.

In this study, the nonlinear vibration theory is applied to the actual model of the forming grinding machine, and the relationship between the grinding force and the vibration is obtained. The establishment of CNC gear form grinding machine grinding mechanics mathematical model and nonlinear vibration system model, respectively, by using the phase plane method of nonlinear vibration equation of the qualitative analysis and the method of multiple scales is used to quantitatively analyze the nonlinear vibration equation; through MATLAB analysis the vibration signal of grinding parameters under different grinding parameters, and then analyzes the voltage signal under different process parameters, finally obtains the relationship between grinding force, process parameters and vibration.

\section{Vibration Model of Gear Form Grinding Machine}

\subsection{Establishment of Vibration Model}

According to the actual operating conditions of CNC gear form grinding machine, the physical model of the nonlinear vibration of grinding wheel is established finally by analyzing the key parts of the grinding machine in the process of machining. Here, ignoring the grinder bed, grinding wheel rack and its affiliated parts influence on vibration, the model is proposed by considering the nonlinear damping and nonlinear stiffness between wheels and workpieces, which is shown in Fig. 1.

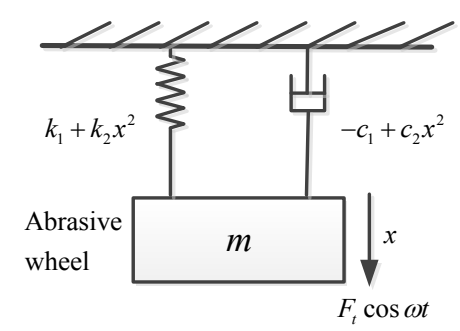

Figure 1. Mechanical mode of workpiece-grinding wheel

where $m$ is the equivalent mass of grinding wheel; $k_{1}$ and $k_{2}$ are the stiffness of stable grinding and the grinding wheel, respectively; $c_{1}$ and $c_{2}$ are the contact damping in the process of grinding and the damping between grinding wheel and grinding wheel, respectively; $F \cos \omega t$ stands for the excitation force of grinding process. Due to the nonlinear damping and nonlinear stiffness between grinding wheel and gear, $k_{1}+k_{2} x^{2}$ is to define the nonlinear stiffness, and $-c_{1}+c_{2} x^{2}$ is to define the nonlinear damping between them, respectively.

Through the established physical model, the nonlinear vibration equation in the grinding process of the gear and grinding wheel is obtained by using kinetics equation:

$$
m \ddot{x}+\left(k_{1}+k_{2} x^{2}\right) x+\left(-c_{1}+c_{2} x^{2}\right) \dot{x}=F \cos \omega t
$$

Substituting the $\sigma=\frac{c_{1}}{m}, \theta=\frac{c_{2}}{m}, \psi=\frac{k_{1}}{m}, \lambda=\frac{k_{2}}{m}, F^{\prime}=\frac{F}{m}$ into the Eq. (1), so it can be expressed as

$$
\ddot{x}+\psi x+\lambda x^{3}-\left(\sigma-\theta x^{2}\right) \dot{x}=F^{\prime} \cos \omega t
$$

The grinding force $F$ can be determined by the following formula according to the reference [18]: 


$$
F=F_{p} \cdot a_{p}^{x} \cdot v_{s}^{y} \cdot v_{w}^{z}
$$

Here $F_{p}$ is the grinding rate constant; $a_{p}$ is the radial grinding depth of grinding wheel; $v_{s}$ is the line speed of grinding wheel, $v_{w}$ is the feed speed of grinding wheel along tooth width direction, $x, y, z$ is an exponent of the expression, respectively.

Multiple scales method is a method of solving nonlinear problems which treats the solution of equation as a function of multiple independent variables.

For a conservative single-degree-of-freedom system:

$$
\ddot{x}+f(x)=0
$$

Suppose the nonlinear function can be expanded by Taylor series at the origin. So the above formula can be written as follows:

$$
\ddot{x}+\sum_{n=1}^{N} a_{n} x^{n}=0
$$

where,

$$
a_{n}=\frac{1}{n !} f^{(\mathrm{n})}(0)
$$

Here $f^{(\mathrm{n})}$ represents the $n$ derivative of the independent variable $x$. At the origin point, the equation can be expressed as: $f(0)=0, f^{(n)}(0)>0$.

$$
T_{n}=\varepsilon^{n} t \quad(n=0,1,2, \cdots)
$$

Here $T_{n}$ is the new independent variable; $\varepsilon$ is the small parameter.

Therefore, the derivative of $t$ turns to the partial derivative of the expansion of $T_{n}$ :

$$
\left\{\begin{array}{l}
\frac{d}{d t}=\frac{d T_{0}}{d t} \frac{\partial}{\partial T_{0}}+\frac{d T_{1}}{d t} \frac{\partial}{\partial T_{1}}+\cdots+\frac{d T_{n}}{d t} \frac{\partial}{\partial T_{n}}=D_{0}+\varepsilon D_{1}+\cdots+\varepsilon^{n} D_{n} \\
\frac{d^{2}}{d t^{2}}=D_{0}^{2}+2 \varepsilon D_{0} D_{1}+\varepsilon^{2}\left(D_{1}^{2}+2 D_{0} D_{2}\right)+\cdots
\end{array}\right.
$$

where, $D_{n}=\frac{\partial}{\partial T_{n}}(n=0,1,2, \cdots)$ is the partial differential operator.

Therefore, the solution of a single degree of freedom nonlinear vibration equation can be expressed as a function of a number of different time scales:

$$
x(t, \varepsilon)=x_{0}\left(T_{0}, T_{1}, T_{2}, \cdots\right)+\varepsilon x_{1}\left(T_{0}, T_{1}, T_{2}, \cdots\right)+\varepsilon^{2} x_{2}\left(T_{0}, T_{1}, T_{2}, \cdots\right)+\cdots+\varepsilon^{n} x_{n}\left(T_{0}, T_{1}, T_{2}, \cdots\right)
$$

The number of the required independent time scale variables depends on the requirements to the firstorder approximate solution in the above formula. Thus, the Eq. (5) can be expressed as follows:

$$
x(t, \varepsilon)=\varepsilon x_{1}\left(T_{0}, T_{1}, T_{2}\right)+\varepsilon^{2} x_{2}\left(T_{0}, T_{1}, T_{2}\right)+\varepsilon^{3} x_{3}\left(T_{0}, T_{1}, T_{2}\right)
$$

Substitute the above Eq. (10) and Eq. (8) into Eq. (5), then the equation of $\varepsilon$-rank can be established. Furthermore, the unknown number $x_{1}, x_{2}, x_{3}, \cdots$ can be approximately calculated.

\subsection{Primary Resonance Solution}

Using the multi-scale method, the primary resonance of the system caused by parametric excitation will be analyzed as follows.

Here $\omega$ is given by

$$
\omega=\sqrt{\psi}+\varepsilon \delta
$$

Substituting Eq. (11) and Eq. (8) into Eq. (2) will give the following solution:

$$
\left\{\begin{array}{l}
D_{0}^{2} x_{0}+\psi x_{0}=0 \\
D_{0}^{2} x_{1}+\psi x_{1}=F^{\prime} \cos \omega t-\lambda x_{0}^{3}+\left(\sigma-\theta x^{2}\right) D_{0} x_{0}-2 D_{0} D_{1} x_{0}
\end{array}\right.
$$

The solution of the equation is defined as: 


$$
x_{0}=A\left(T_{1}\right) \exp \left(i \omega T_{0}\right)+c
$$

where $A$ is the complex function of $T_{1}$ and $A=\frac{1}{2} b\left(T_{1}\right) \exp \left[i \varphi\left(T_{1}\right)\right], c$ is the conjugate function of $A\left(T_{1}\right) \exp \left(i \omega T_{0}\right)$.

Substituting $x_{0}$ into Eq. (12):

$$
\begin{aligned}
& D_{0}^{2} x_{1}+\psi x_{1}=\left(-3 \lambda A^{2} \bar{A}-2 i \sqrt{\psi} D_{1} A\right) \exp (i \sqrt{\psi} t)+\left(\sigma i \sqrt{\psi} A-2 \theta A^{2} \bar{A} \sqrt{\psi}\right) \exp (i \sqrt{\psi} t) \\
& +1 / 2 F^{\prime} \exp (i \omega t)-\left(\theta A^{3}+\lambda A^{3}\right) \exp (3 i \sqrt{\psi} t)+c
\end{aligned}
$$

To eliminate the secular term, the coefficient of $\exp \left(i \sqrt{\psi} T_{0}\right)$ in Eq. (14) should be defined to zero. So we have

$$
1 / 2 F^{\prime} \exp (i \omega t)-3 \lambda A^{2} \bar{A}-2 i \sqrt{\psi} D_{1} A+\sigma i \sqrt{\psi} A-2 \theta A^{2} \bar{A} \sqrt{\psi}=0
$$

Combined with the Eq. (15), the real and imaginary part of the equation can be separated as follows:

$$
\left\{\begin{array}{l}
\left(\sqrt{\psi} b \varphi^{\prime}-\frac{3 \lambda b^{3}}{8}\right) \cos \varphi+\left(b^{\prime} \sqrt{\psi}-\frac{\sigma b \sqrt{\psi}}{2}+\frac{\theta b^{3} \sqrt{\psi}}{4}\right) \sin \varphi+\frac{1}{2} F^{\prime} \cos \omega t=0 \\
\left(\sqrt{\psi} b \varphi^{\prime}-\frac{3 \lambda b^{2}}{8}\right) \sin \varphi-\left(b^{\prime} \sqrt{\psi}-\frac{\sigma b \sqrt{\psi}}{2}+\frac{\theta b^{3} \sqrt{\psi}}{4}\right) \cos \varphi+\frac{1}{2} F^{\prime} \cos \omega t=0
\end{array}\right.
$$

According to Eq. (16), we have

Furthermore, we have

$$
\left\{\begin{array}{l}
b^{\prime} \sqrt{\psi}-\frac{\sigma b \sqrt{\psi}}{2}+\frac{\theta b^{3} \sqrt{\psi}}{8}-\frac{1}{2} F^{\prime} \sin (\omega t-\varphi)=0 \\
\sqrt{\psi} b \varphi^{\prime}-\frac{3}{8} \lambda b^{3}+\frac{1}{2} F^{\prime} \cos (\omega t-\varphi)=0
\end{array}\right.
$$

$$
\left\{\begin{array}{l}
b^{\prime}=\frac{\sigma b}{2}+\frac{\theta b^{3}}{8}+\frac{F^{\prime} \sin (\omega t-\varphi)}{2 \sqrt{\psi}} \\
b \varphi^{\prime}=\frac{3 \lambda b^{3}}{8 \sqrt{\psi}}-\frac{F^{\prime} \cos (\omega t-\varphi)}{2 \sqrt{\psi}}
\end{array}\right.
$$

Let $\varphi_{1}=\omega t-\varphi$, and the main resonance first-order approximate solution can be got as follows:

$$
x_{1}=b \cos \left(\sqrt{\psi} t+\varphi_{1}\right)+o(\varepsilon)
$$

\section{Gear Form Grinding Machine Vibration Test}

In order to understand the main vibration source and the vibration mechanism of the grinding machine, the experiment platform is set up for exploring the influence of grinding process parameters on vibration characteristics. For the study of vibration characteristics and stability of grinding process, it is the best way to select components of acceleration vibration signals and grinding forcer signals.

Based on the experimental purpose, the experimental scheme is built. The experimental scheme is to measure the electric current signal of electric spindle on voltage test platform and the vibration signal of the numerical control servo axis on vibration test platform by changing the grinding parameters respectively. The vibration test platform is set up as shown in Fig. 2. 


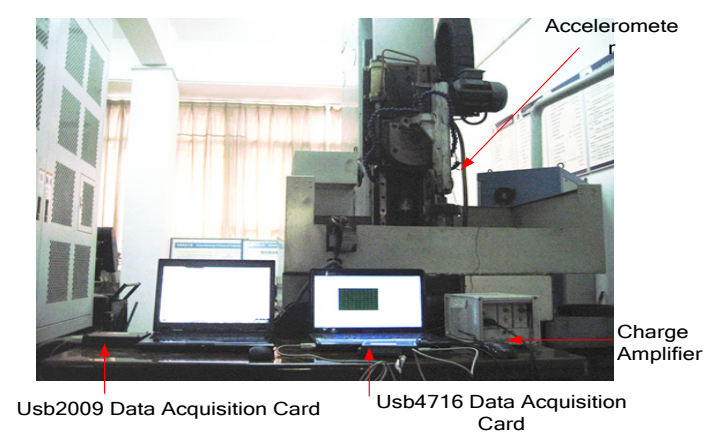

Figure 2. Acquisition test bench of CNC gear form grinding machine

The test platform is built on YK7660 gear form grinding machine. Suppose the speed of the grinder spindle motor is $2840 \mathrm{r} / \mathrm{min}$, the rated current is $3.43 \mathrm{~A}$, and the frequency of the inverter is $50 \mathrm{~Hz}$. The gear and grinding wheel parameters of the grinding process are shown in Table 1. The process parameters are shown in Table 2.

Table 1. Gear and grinding wheel parameters

\begin{tabular}{llll}
\hline Items & & \multicolumn{1}{l}{ Gear } \\
\hline$(A)$ Gear data & & & \\
Teeth number & $z$ & - & 26 \\
Normal module & $m_{n}$ & $\mathrm{~mm}$ & 5 \\
Pressure angle & $a_{n}$ & $\mathrm{deg}$ & 20 \\
Helix angle & $\beta$ & $\mathrm{deg}$ & 11.478 \\
Face width & $b$ & $\mathrm{~mm}$ & 53 \\
Coefficient of profile shift & $x_{n}$ & - & -0.032 \\
(B) Wheel data & & & \\
Outer diameter & $d_{w}$ & $\mathrm{~mm}$ & 300.000 \\
Width & $w_{w}$ & $\mathrm{~mm}$ & 60.000 \\
(C) Machine settings & & & \\
Setting angle of the wheel & $\gamma_{m}$ & $\mathrm{deg}$ & 11.478 \\
Center distance & $E_{t}$ & $\mathrm{~mm}$ & 210.077 \\
Axial movement & $L_{t}$ & $\mathrm{~mm}$ & $326.647 \varphi_{1}$ \\
\hline
\end{tabular}

Table 2. Process parameters

\begin{tabular}{llll}
\hline Serial number & $v_{\mathrm{c}}(\mathrm{m} / \mathrm{s})$ & $v_{\text {u. }}(\mathrm{mm} / \mathrm{s})$ & $a_{\mathrm{o}}(\mathrm{mm})$ \\
\hline 1 & 16.8 & 13.0 & 0.01 \\
2 & 16.8 & 18.8 & 0.01 \\
3 & 18.8 & 6.93 & 0.01 \\
4 & 18.8 & 6.93 & 0.02 \\
\hline
\end{tabular}

\section{Analysis of Vibration Characteristics}

\subsection{Vibration Analysis under Different Feed Speeds}

The workpiece feed speed is one of the grinding process parameters. Fig. 3 expresses the vibration of grinding wheel rack with different workpiece feed speed $v_{w}$ under the constant linear velocity $v_{s}=16.8 \mathrm{~m} / \mathrm{s}$, and the grinding depth $a_{p}=0.01 \mathrm{~mm}$. As shown in Fig. 3, the acceleration sensor collects six groups of vibration data under the constant grinding wheel linear velocity $v_{s}$ and grinding depth $a_{p}$. 
When the workpiece feed speed $v_{w}$ is $13 \mathrm{~mm} / \mathrm{s}$, the sensor respectively collects vibration signals of $X, Y$, and $Z$ three shafts labeled as Fig. 3(a), Fig. 3(c) and Fig. 3(e), respectively. When the workpiece feed speed $v_{w}$ is $18.9 \mathrm{~mm} / \mathrm{s}$, the sensor collects vibration signals of $X, Y$, and $Z$ three shafts labeled as Fig. 3(b), Fig. 3(d) and Fig. 3(f), respectively.

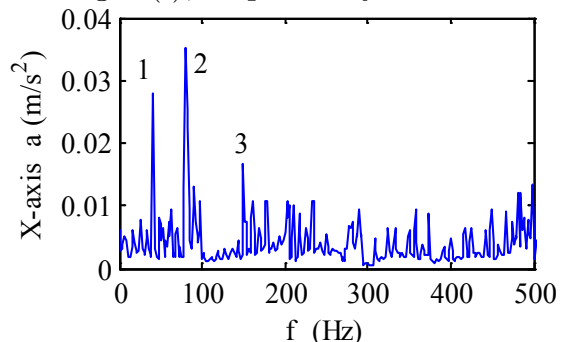

(a) $v_{w}=13 \mathrm{~mm} / \mathrm{s}$

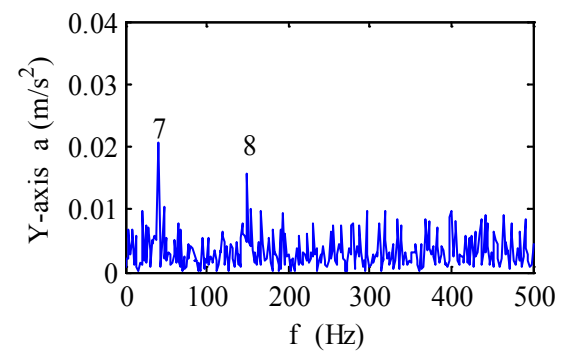

(c) $v_{w}=13 \mathrm{~mm} / \mathrm{s}$

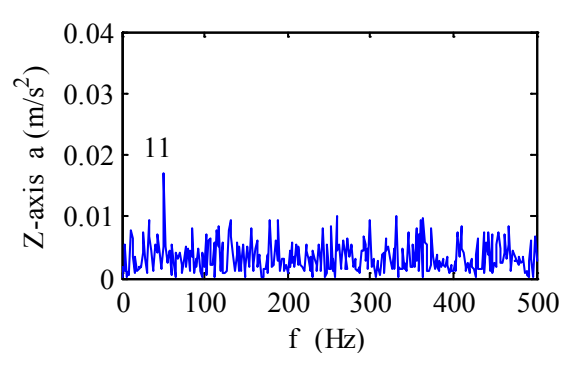

(e) $v_{w}=13 \mathrm{~mm} / \mathrm{s}$

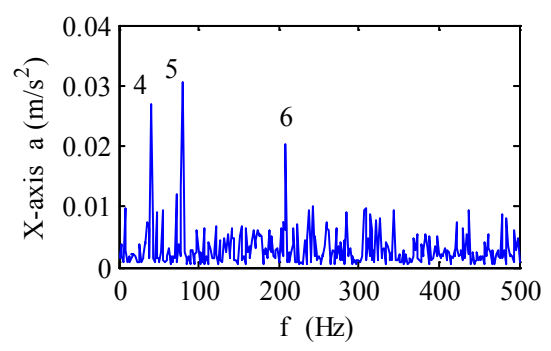

(b) $v_{w}=18.8 \mathrm{~mm} / \mathrm{s}$

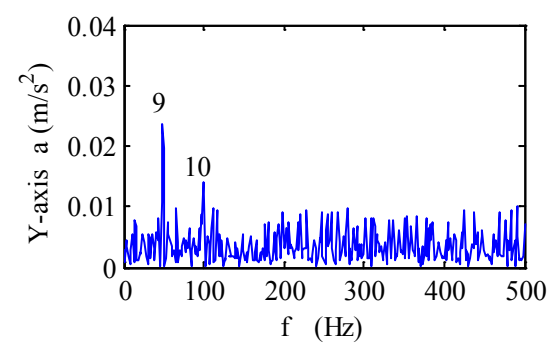

(d) $v_{w}=18.8 \mathrm{~mm} / \mathrm{s}$

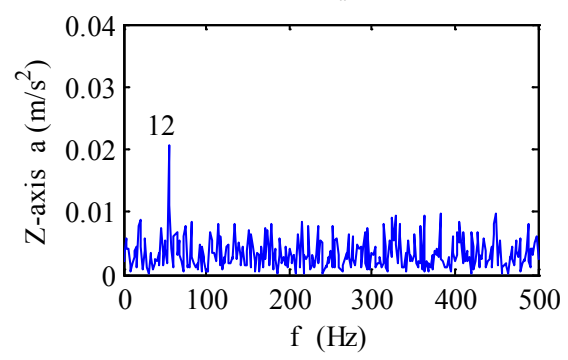

(f) $v_{w}=18.8 \mathrm{~mm} / \mathrm{s}$

Figure 3. Acceleration vibration signal spectrum in different workpiece speed.

In Fig. 3(a) and Fig. 3(b), the frequency at spectrum peak labeled as 1 and 4 is $40 \mathrm{~Hz}$, which is consistent with the spindle rotation frequency when the linear velocity is $16.8 \mathrm{~m} / \mathrm{s}$. Similarly, the frequency at spectrum peak labeled as 2 and 5 is about $80 \mathrm{~Hz}$, which is in multiple relations to the spindle rotation frequency. The results show that the previous supposition about the vibration source caused by spindle rotation is right. Through the horizontal comparison of different frequencies peak, the result shows that the larger the feed speed is, the greater impact of the spindle for grinding will be. The frequency of label 3 and 6 at spectrum peak is $150 \mathrm{~Hz}$ and $210 \mathrm{~Hz}$, respectively. When the speed $v_{w}$ is $16.8 \mathrm{~m} / \mathrm{s}$, the frequency of spindle rotation is $38 \mathrm{~Hz}$ which is roughly equal to the third order and fourth order of the frequency of the grinding wheel frame. The results show that the high order mode vibration is occurred in these two places, and with the feed speed increasing, the order of higher order modes is increasing. The reason why high order mode vibration occurs is that different frequency of forced vibration is produced by grinding wheel drive motor. The vibration components of these frequency are not all expressed as flutter, and part of the frequency is close to the inherent frequency of grinding wheel rack. Consequently, the vibration of high order mode occurs. As known the vibration source of label 7 and 9 is also caused by spindle rotation. The possibility of higher order mode vibration in label 8 is higher. The frequency of label 11 and 12 is about $38 \mathrm{~Hz}$, whereas, the peak in other frequency ranges does not appear which explains that the spindle rotation has less effect on the $Z$ axis vibration. Longitudinal, from Fig. 3(a), Fig. 3(c) and Fig. 3(e), the source of vibration is mainly caused by the 
spindle rotation and forced vibration of motor. In addition, if there are other vibration sources, it will be the self-excited vibration which is caused by grinding force. As shown from the amplitude, the forced vibration produced by motor makes the most intense influence on $X$ axis, the second is the $Y$ axis, $Z$ axis is the weakest. Using the same theory to analyze the Fig. 3(b), Fig. 3(d) and Fig. 3(f), the same conclusion can be got.

All the above results show that the source of vibration produced by grinding wheel at different workpiece feed speeds is mainly caused by spindle rotation, and the spindle rotation has the greatest influence on the $X$ axis, the second is the $Y$ axis, $Z$ axis is the weakest. Moreover, with the increasing of workpiece feed speed, the effect of spindle rotation on grinding is increasing.

\subsection{Vibration Analysis under Different Grinding Depths}

Gear grinding usually is the last procedure of gear precision manufacturing. This grinding $\operatorname{depth} a_{p}$ in experiment is $0.01 \mathrm{~mm}$ or $0.02 \mathrm{~mm}$, respectively. When the linear velocity of grinding wheel $v_{s}=18.8 \mathrm{~m} / \mathrm{s}$ and the workpiece feed speed $v_{w}=6.93 \mathrm{~mm} / \mathrm{s}$ are constant, the grinding force changes with the increasing of the grinding depth. Therefore, the relative position between grinding wheels and workpieces will be influenced by the grinding force which would result in the occurrence of self-excited vibration. Fig. 4 reflects the vibration of grinding wheel rack on different grinding depths.

As shown in Fig. 4, the acceleration sensors also collect six groups of vibration data. When the grinding depth $a_{p}$ is $0.01 \mathrm{~mm}$, the sensors collect vibration signals of $X, Y$, and $Z$ three shafts labeled as Fig. 4(a), Fig. 4(c) and Fig. 4(e), respectively. When the grinding depth $a_{p}$ is $0.02 \mathrm{~mm}$, the sensors collect vibration signals of $X, Y$, and $Z$ three shafts labeled as Fig. 4(b), Fig. 4(d) and Fig. 4(f), respectively. The frequency of label 1 and 3 respectively is $45 \mathrm{~Hz}$ and $40 \mathrm{~Hz}$, and it is similar to the rotation frequency of the spindle when grinding wheel line speed is $18.8 \mathrm{~m} / \mathrm{s}$. The results show that the vibration sources of the two are caused by the rotation of the main shaft. Meanwhile, the bigger the grinding depth is, the greater the effect of spindle rotation on grinding will be. The frequency labeled as 2 and 4 at spectrum peak is about $80 \mathrm{~Hz}$, which is in multiple relations to the spindle rotation frequency when the linear velocity of grinding wheel is $18.8 \mathrm{~m} / \mathrm{s}$. The results show that the two sources of vibration are still caused by spindle rotation. From Fig. 4(a), Fig. 4(c) and Fig. 4(e), the vibration source of $X$ axis, $Y$ axis and $Z$ axis is caused by the spindle rotation. However, the amplitude of the label 2 is larger than that of the label 6 and 10, which explains the spindle rotation has the greatest influence on the $X$ axis, the second is the $Y$ axis, $Z$ axis is the weakest.

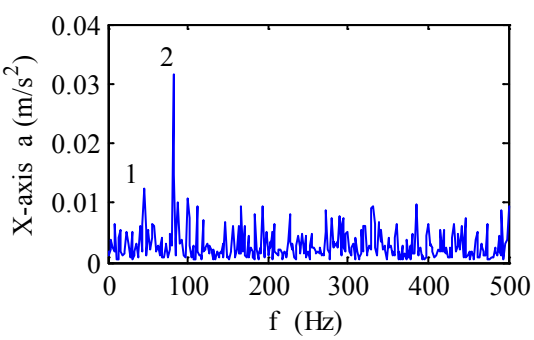

(a) $a_{p}=0.01 \mathrm{~mm}$

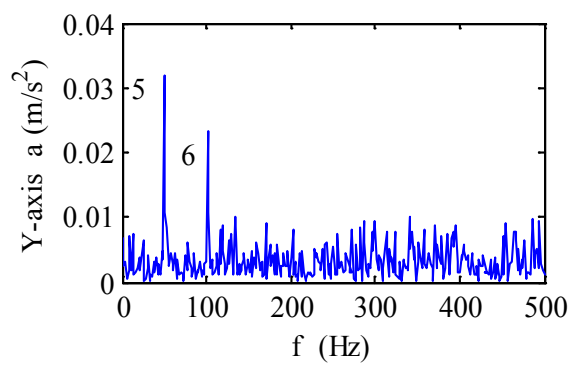

(c) $a_{p}=0.01 \mathrm{~mm}$

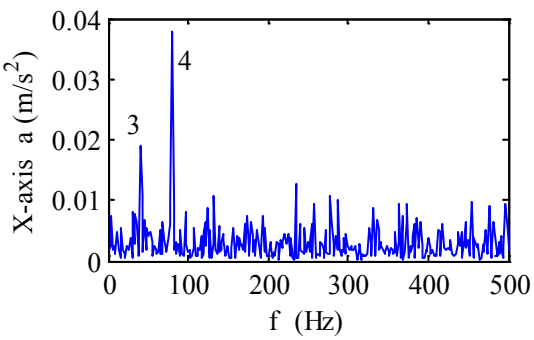

(b) $a_{p}=0.02 \mathrm{~mm}$

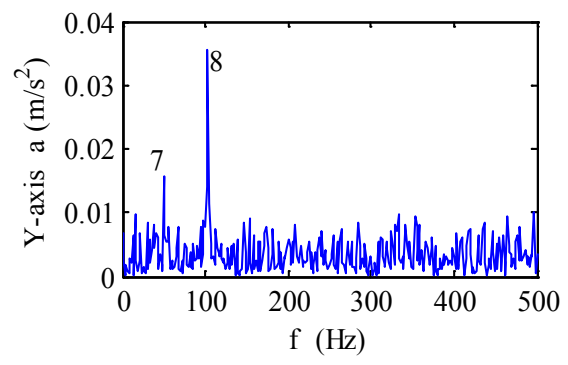

(d) $a_{p}=0.02 \mathrm{~mm}$ 


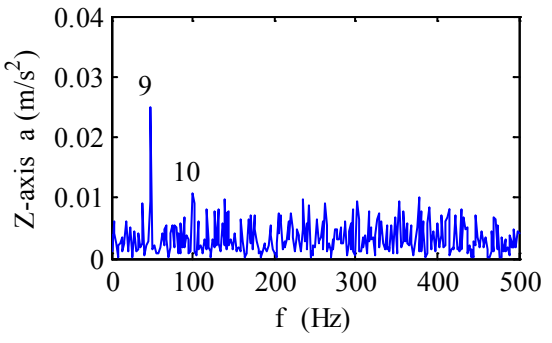

(e) $a_{p}=0.01 \mathrm{~mm}$

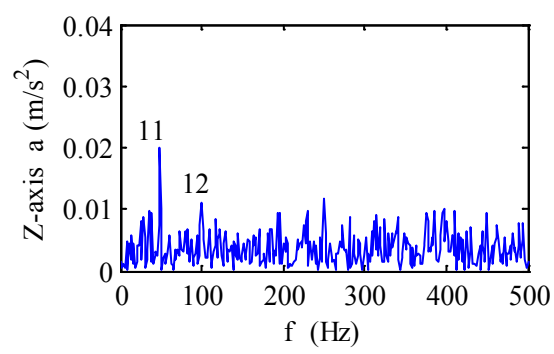

(f) $a_{p}=0.02 \mathrm{~mm}$

Figure 4. Acceleration vibration signal spectrum in different grinding depth.

\section{Conclusions}

Based on the condition of this experiment on the gear form grinding machine, this paper has collected the grinding force signal and acceleration vibration signal, which is one useful way to evaluate the vibration characteristics of gear form grinding. The following conclusions are obtained in this experiment:

1) Grinding force is controlled by grinding process parameters. The process parameters also influence the grinding process, so the grinding force is an important parameter in the grinding process. The grinding force is closely linked with the generation of the self-excited vibration. However, the generation of the self-excited vibration will influence the grinding process. When the vibration is serious, it will cause the grind to be unable to continue.

2) Under the condition of constant linear velocity of grinding wheel and grinding depth, the increasing of workpiece feed speed does not make the grinding force get bigger. The reason why this situation does not appear is likely that the feed rate is smaller. Under the condition of constant linear velocity and workpiece feed speed, with the increasing of grinding depth, the grinding force gets bigger and bigger, but it does not occur self-excited vibration. The reasons may be that the low-speed stability effect occurs. Through the acquisition and comparative analysis of vibration signal under the non-grinding condition, the result shows that under the grinding condition, the main source of vibration signal is generated by the rotating spindle.

3) Changing the grinding process parameters, the experimental results show that vibration on the $X$ axis is the biggest, and $Z$ axis is the smallest. With the increasing of feed speed and grinding depth of the workpiece, the influence of the forced vibration caused by each shaft becomes larger.

Acknowledgement. The authors are grateful to the National Natural Science Foundation of China (Grant No.51575160), and the scientific and technological project of Henan Province (Grant No. 172102210038) and the Henan Natural Science Foundation (Grant No. 182300410229), for their financial support.

\section{References}

1. Litvin F. L., Fuentes A. Gear Geometry and Applied Theory, chapters 17 and 28, Cambridge University Press, New York, NY, USA, 2nd edition, 2004.

2. Litvin F. L., Gonzalez-Perez, Fuentes A., et al. Topology of modified surfaces of involute helical gears with line contact developed for improvement of bearing contact, reduction of transmission errors, and stress analysis. Mathematical and Computer Modelling, vol. 42 no. 9-10, pp. 1063-1078, 2005.

3. Shih Y. P., Fong Z. H. Flank correction for spiral bevel and hypoid gears on a six-axis CNC hypoid gear generator. Journal of Mechanical Design, vol. 130, no. 6, pp. 876-877, 2008.

4. Zhang Y. Y., Dong M. Analysis on machining technologies of hardened gear. Mechanical Management and Development, vol. 24, no. 4, pp. 95-96, 2009. 
5. Deng X. Z., Zhang Y. Z. Present research situation and technological problem need to solve of the accuracy control of cycloidal-pinwheel transmission used on RV reducer. Mechanical Transmission, vol. 39, no. 2, pp.162$165,2015$.

6. Li Z., Mao K. The tooth profile modification in gear manufacture. Applied Mechanics and Materials, vol.10 no. 12, pp. 317-321, 2008.

7. Eritenel T., Parker R. G. Nonlinear vibration of gears with tooth surface modifications. ASME Journal of Vibration and Acoustics, vol. 135, no. 051005, pp. 1-11, 2013.

8. Guo X. D., Jiang J. K. Look from CCMT2010 new trends of gear grinding machine market development. The World's Manufacturing Technology and Equipment Market, vol.12, no. 5, pp. 42-44, 2010.

9. Song X. L. Wheel Frame Optimal Design of CNC Forming Grinding Machine based on ABAQUS. Journal of Mechanical Transmission, vol. 37, no. 2, pp.1-3, 2013.

10. Orynski F., Pawtowski W. The influence of grinding process on forced vibration damping in headstock of grinding wheel of cylindrical-grinder. International Journal of Machine Tools 8 Manufacture, vol. 39, no. 2, pp. 229-235,1999.

11.Krajnik P., Kopac J., Sluga A. Design of grinding factors based on response surface methodology. Journal of Materials Processing Technology, vol. 162, no. 20, pp. 629-636, 2005.

12.Toenshoff H. K., Spinting W. Machining of holes developments in drilling technology. Annals of the CIRP, Vol. 43, no. 2, pp. 551-561, 1994.

13.Toenshoff H. K., Peters J. Modeling and simulation of grinding processes. Annals of CIRP, vol. 41, no. 2, pp. 677-688, 1992.

14.Hashimoto F., Kanai A., Miyashita M. Growing mechanism of chatter vibration in grinding processes and chatter stabilization index of grinding wheel. Journal of the Japan Society for Precision Engineering, vol. 33, no.1, pp. 715-721,1983.

15.Milashita M., Hashimoto F., Kanai, A. Diagram for selecting chatter free conditions of centerless grinding. Annals CIRP, vol. 31, no. 1, pp. 221-223, 1982.

16.Inasaki I., Tonou K., Yonestu S. Regenerative chatter in cylindrical plunge grinding. Bulletin of the JSME, vol. 20, no.150, pp. 1648-1654, 1977.

17.Weck M., Alldieck J. The originating mechanisms of wheel regenerative grinding vibration by alternating wheel speed. CIRP Annals-Manufacturing Technology, vol. 38, no. 1, pp. 231-234, 1986.

18. Matsubara T., Mizumoto H., Yamamoto H. Experimental analysis of work regenerative chatter in plunge grinding [J]. Bulletin of the Japan Society of Precision Engineering, vol.21, no.1, pp.33-37, 1987.

19.Ebrahimi M., Whalley R. Machine tool syntheses in virtual machining. International Journal of Materials 8 Product Technology, vol.13, no.3, pp.301-312, 1998.

20.Wang H. L., Deng X. Z. Dynamic assessment of vibration of tooth modification gearbox using grey bootstrap. Shock and Vibration, vol. 2015, Article ID 204609, 8 pages, 2015.

21.Zhu P. F. Experimental research on tangential grinding force of gear form grinding. Diamond E Abrasives Engineering, vol. 33, no.4, pp. 74-77, 2013. 